\title{
Evolving presentation of leg cramps in a child with nephrotic syndrome: Questions
}

\author{
Amirtha V. Chinnadurai ${ }^{1}$ • Olivera Marsenic ${ }^{1}$ \\ Received: 12 December 2016/Revised: 19 December 2016/Accepted: 21 December 2016 /Published online: 23 February 2017 \\ (C) IPNA 2017
}

Keywords Child $\cdot$ Nephrotic syndrome $\cdot$ Leg cramps

\section{Case}

An 8-year-old African-American boy was diagnosed with nephrotic syndrome 3 months prior to presentation. He was noted to have proteinuria (4+ on urine dipstick) for four consecutive days while on prednisone wean and was restarted on prednisone $60 \mathrm{mg}$ daily. The next day, he started experiencing intermittent cramps in his fingers and right lower leg. He presented to the emergency room (ER) 2 days later for evaluation of worsening right leg cramps. The cramps were described as intermittent, severe and not relieved with changes in position. Pain improved after administration of acetaminophen. Initial evaluation in the ER was unremarkable, and he was discharged home, but returned to the ER the same night after being woken up from sleep with severe and persistent right lower leg cramps with inability to bear weight without associated paresthesia or numbness. There was no history of trauma or acute illness, and review of systems was otherwise negative.

Apart from nephrotic syndrome, his past medical history was unremarkable. There was no significant family history of renal, autoimmune, or blood clotting disorders. Social history was unremarkable. There was no suspicion for non-accidental trauma.

The answers to these questions can be found at http://dx.doi.org/10.1007/ s00467-017-3606-7.

Amirtha V. Chinnadurai

amirtha.chinnadurai@yale.edu

Yale-New Haven Hospital, New Haven, CT, USA
Physical exam was remarkable for significant distress secondary to the leg pain. The right lower extremity was pale and cool on palpation with prolonged capillary refill time of 4-5 s, in comparison to the left. The right popliteal, dorsalis pedis, and posterior tibial pulses, although present, were diminished compared to the left. Sensation was intact. He had mild periorbital swelling, but there was no lower extremity edema noted. His vital signs were: temperature $98.3 \mathrm{~F}$, blood pressure $110 / 60 \mathrm{mmHg}$, heart rate 120 beats/min, and respiratory rate $20 /$ min, oxygen saturation of $98 \%$.

Laboratory investigations showed serum creatinine of $0.8 \mathrm{mg} /$ $\mathrm{dl}$, glomerular filtration rate (GFR) of $70.6 \mathrm{ml} / \mathrm{min} / 1.73 \mathrm{~m}^{2}$ (bedside Schwartz formula), serum sodium $132 \mathrm{mmol} / \mathrm{l}$, serum calcium $7.6 \mathrm{mg} / \mathrm{dl}$, serum albumin $1.4 \mathrm{~g} / \mathrm{dl}$. Other electrolytes including magnesium and phosphate were within the normal range. Complete blood count showed white blood cell count $5600 / \mu 1$, hematocrit $39 \%$, hemoglobin $13.6 \mathrm{~g} / \mathrm{dl}$, platelet count 273,000/ $\mu$ l. Urine protein/creatinine ratio was $29.5 \mathrm{mg} / \mathrm{mg}$. Plain radiographs of the lower extremity did not reveal fractures, dislocations, joint effusions, or signs of osteomyelitis.

\section{Questions}

1. What is the differential diagnosis?

2. What are the next diagnostic steps?

3. What is the management for this condition? 